\title{
Experience-dependent plasticity in the developing brain
}

\author{
Irini Skaliora*
}

University Laboratory of Physiology, Oxford University, Oxford, OX1 3PT, UK

\begin{abstract}
The extent to which individual experiences can shape our cognitive and emotional behaviour has been an issue of intense debate and study for centuries, and the topic takes on different forms depending on the field of study. Any form of behaviour will depend on the existence of functional connections in the brain, linking inputs and outputs. If these connections are damaged, or in any way silenced or suppressed, the related behaviour will be impaired, or modified accordingly. A large part of ontogenesis is devoted to the formation of such connections within and between different brain structures. Whereas the initial formation of connections is largely determined by chemical guidance factors and thus genetically specified, the ensuing neuronal circuits remain 'plastic' or modifiable for a prolonged period and, in some cases, throughout life. In this paper, I define notions of plasticity from a neurobiological perspective. I use the example of experience-dependent plasticity in multisensory convergence and integration in the brain, in order to illustrate the extent and limitations of the role of experience in sculpting neuronal circuits. I then describe briefly the various mechanisms that are thought to underlie experience-dependent changes in brain circuits during early development. (C) 2002 Elsevier Science B.V. All rights reserved.
\end{abstract}

Keywords: Neuronal plasticity; Multisensory integration; Development

\section{Introduction}

The centuries-old debate on 'Nature vs. Nurture' is one that has been particularly susceptible to social and cultural trends and continues to generate passionate arguments. In

Abbreviations: AMPA, alpha-amino-3-hydroxy-5-methyl-4-isoxazolepropionate; ITD, interaural time difference; ILD, interaural level difference; ICC, inferior colliculus; ICX, external nucleus of the inferior colliculus; $\mathrm{nBIC}$, nucleus of the brachium of the inferior colliculus; NMDA, $N$-methyl-D-aspartate; SC, superior colliculus.

* Tel.: +44-1865-272438; fax: +44-1865-282498.

E-mail address: irini.skaliora@physiol.ox.ac.uk (I. Skaliora). 
modern neuroscience, it is more appropriate to speak of specificity vs. plasticity of neuronal connections and the goal of researchers is to reveal how the two interact in order to produce an organism that is adapted to its environment.

Any type of behaviour will depend on the existence of precisely ordered functional circuits in the brain. These connections need to be exquisitely specific and precise and failure to be so will result in deficits of varying degrees. The study of behaviour is thus inexorably linked with the study of the neural circuits that mediate it: how they form and to what extent they can be modified subsequently.

During development, neurons are generated in dedicated regions of the brain, from where they subsequently have to migrate to their final destinations. They also need to extend neuronal processes, one of which, the axon, must often travel long distances to locate the target and make synaptic connections with the appropriate type of cells. All this basic wiring of the brain is genetically specified: neurons are programmed to express particular combinations of molecules, which interact in stereotyped ways with molecules on other cells and in the extracellular environment [1]. As a result of these interactions, neurons and their axons are guided to the right part of the brain. Such mechanisms give rise to an orderly, stereotyped and relatively stable set of connections. This is specificity, and it is an essential requirement for the proper functioning of the brain.

These connections, however, which are still crude and usually overabundant, are further elaborated and refined by neuronal activity, initially generated spontaneously and subsequently driven by sensory experience [2]. This is plasticity, and it allows brains, and thus behaviour, to adapt to features of the environment as experienced by the individual. In this paper, I illustrate the principles of specificity and plasticity in the context of multisensory integration in the superior colliculus (SC) and discuss the possible mechanisms that are thought to underlie the initial establishment and subsequent reorganization of neuronal connections.

\section{Neuronal circuits in the superior colliculus}

The mammalian SC, or its homologous tectum in birds and reptiles, is located in the midbrain, and is a crucial site for multisensory convergence and integration. In this layered structure, signals from different senses converge and are combined in order to produce coherent representations of the external world and guide orientation behaviour. The superficial layers are involved exclusively in visual processing and are innervated by fibres from the retina and visual cortex. In contrast, the deeper layers receive multiple types of inputs, including: (i) visual information relayed through the superficial layers; (ii) auditory input, the main source of which comes from the nucleus of the brachium of the inferior colliculus (nBIC); (iii) somatosensory input. Cells in the deeper layers also receive feedback projections from higher cortical areas. Many of these cells have motor functions and control head and eye movements that result in visual fixation of the activating stimulus, irrespective of its modality.

An important feature of the SC is that its layers contain topographic (or spatial) maps of the different modalities. In other words, cells are spatially arranged so that neighbouring cells signal adjacent sensory stimuli-in an orderly way to cover the entire extent of 


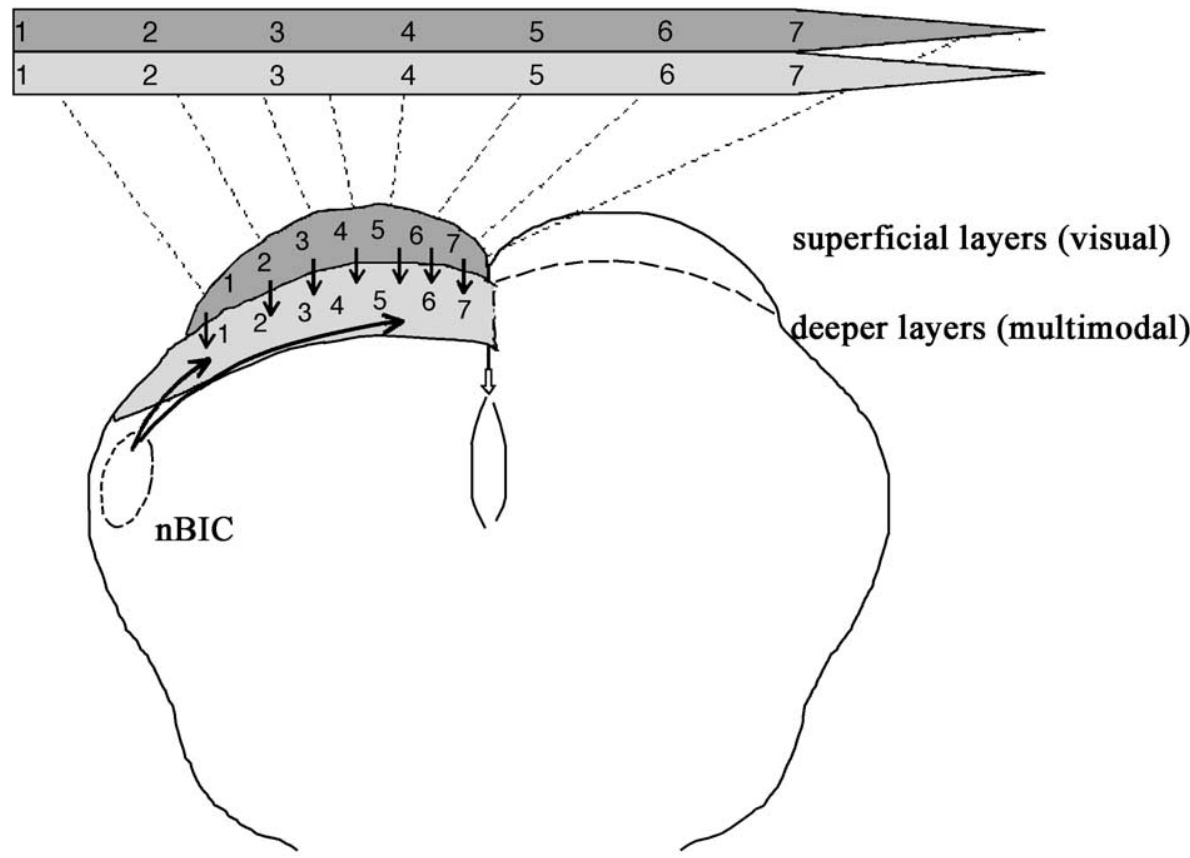

Fig. 1. Schematic illustration of a cross section of the superior colliculus (SC). The two elongated numbered boxes at the top illustrate the orderly topographic representation of visual and auditory space. The superficial SC layers (dark grey) receive exclusively visual inputs and contain a topographic map of visual space (as indicated schematically by the correspondence of the numbers). The deeper layers (light grey) receive inputs from several modalities, including visual inputs through the superficial layers (vertical arrows) and auditory inputs from the nucleus of the brachium of the inferior colliculus (nBIC) and also contain a topographic map of auditory space. In the adult animal, the visual and auditory maps of space are aligned, as indicated by the matching numbers.

sensory space (Fig. 1). This topographic organization is very important for perception and for the coherent representation of the external world in our brains, and is found in all sensory systems. Furthermore, a rather unique feature of the SC is that these maps are aligned to each other (Fig. 1). So that, if a bimodal cell in the deeper multisensory layers responds to visual stimulus from a given region in space, it will also respond to an auditory signal from that same region in space. Alignment of sensory maps is essential for the interpretation of the multisensory interactions manifested by SC neurons, which depend on the spatial and temporal relationship between different stimuli [3].

\section{Development of visual and auditory maps in the superior colliculus}

So how are these maps formed and maintained? The visual map is a typical 'projection map', where visual space is naturally transformed into two-dimensional retinal space - and its topography (i.e. the neighbouring relations of the cells) is preserved in the projection patterns to subsequent brain structures. This is a case of a genetically specified topographic 
map, as it is largely established before the onset of vision and thus independent of experience-dependent activity.

In contrast, auditory space is never directly reflected in the arrays of auditory receptors, which instead, systematically code the frequency components of the auditory stimulus. Thus, the extremely important function of locating sounds in space is computed from several cues, especially interaural differences in time (ITD) and level (ILD), but also spectral cues related to the filtering properties of the ear that vary with direction of the sound source [4]. These cues are used to form a computational map of auditory space, which exists in its most refined form in the deep layers of the SC. Thus, a topographic map of auditory space has to be computed, rather than simply relayed, and then it has to be matched to the visual template. We already know that this is a process which occurs during postnatal development, after the animal becomes able to hear and see, and that it occurs in three stages: (i) cells in the deep layers become acoustically active, but there is no topographically ordered map; (ii) a map gradually emerges (cells respond selectively to sounds coming from particular locations and not from others); and (iii) the map is aligned to the visual coordinates [5].

We also know that this map alignment is a process that depends on experience and requires coordinated sensory-driven electrical activity. For instance, if the visual map is experimentally disturbed, by prism rearing or by surgically deviating the eyes, then the visual and auditory maps will become misaligned. In other words, visual and auditory signals from the same region in space will now appear to originate from different regions. Under these circumstances, and provided the manipulation occurs early in life, the auditory space map is gradually recalibrated so that it becomes aligned with the modified visual coordinates [5]. This is a gradual process that involves two components: (i) acquisition of responses that are adaptive with the prisms in place (learned responses) and (ii) elimination of the responses that were previously effective but no longer appropriate with the prisms in place (normal responses) [6]. This is one of the clearest examples of the adaptive nature of experience-dependent plasticity: it allows the alignment of the maps to be established and maintained in spite of individual differences in the size, shape and relative positions of the sense organs.

Importantly, the range of such adaptive modifications depends on the age of the animal. Whereas in young animals, large changes in spatial tuning are induced readily by manipulation of experience, the same manipulations in the adult will cause little if any adjustment of the auditory space map [7]. The period during development when large changes occur as a result of experience is referred to as the sensitive or critical period, for this particular experience.

Even more remarkably, however, experience during the critical period can sometimes leave a permanent 'trace of learning' in the brain and allow an enhanced capacity for adaptive adjustments. Studies with owls have shown that adults that were reared with prisms, but in which normal visual experience was subsequently restored, were able to recover the normal spatial tuning. In addition, re-exposure to prisms as adults resulted in the re-expression of the adaptive responses that were learned during the sensitive period [8]. This enhanced capacity for plasticity persists well into adulthood, but is restricted to the previously learned associations. In other words, prismatic displacement of a different magnitude or direction in the adult will NOT lead to an adaptive readjustment of spatial 
tuning of that different magnitude or direction. Thus, a learning experience during the critical period seems to leave an enduring trace in the corresponding pathway that enables substantial functional plasticity in the adults. This trace of learning specifically reflects the range of tuning states acquired during the sensitive period. Taken together such results indicate that wide-ranging neonatal experience may extend the potential of the adult brain.

The ultimate goal is to understand where these adaptive adjustments occur and what is the underlying mechanism. The answers to such questions are still incomplete and, at least to some extent, species-dependent. Here, I will briefly summarize the current understanding, pointing out important differences between species or discrepancies in the literature.

\section{Site of plasticity}

The adaptive adjustments observed at the level of the SC or tectum could in principle have occurred anywhere leading to that structure. In owls, the main site of plasticity seems to be the external nucleus of the inferior colliculus (ICX), which is the primary source of auditory input to the tectum and the first structure to contain a map of auditory space. Whereas in the inferior colliculus (ICC) of prism-reared owls, the ITD maps were entirely normal, in the ICX, they had shifted to the direction that would account for the adaptive shift in behaviour [9]. In contrast, in mammals, the main site of plasticity is likely to be within the SC itself. Partial neonatal lesions of the superficial visual layers of the ferret SC were found to disrupt the development of auditory space map in the deep layers below the lesion, while responses remained normal in the un-operated SC [10].

\section{Mechanisms of plasticity}

The cellular and molecular mechanisms underlying such adaptive plasticity are still poorly understood. Anatomical studies in owls have shown that in prism-reared animals, the projections from the inferior colliculus to the ICX are re-organized in a way that can account for the shift to the adaptive direction [11]. In these animals, each ICX received the normal ICC inputs, but also received additional inputs from regions in the ICC with spatial selectivity that matched the shifted selectivity of ICX neurons. Therefore, it seems that at least in this system, this novel anatomical input could account for the adaptive shift in the physiology of the neurons and the behaviour of the animal. In addition, the persistence of the normal ICC-to-ICX projection in prism-reared animals can also account for the ability to recover a normal map of ITD in the tectum throughout the lifetime of the animal [6]. Whether this new anatomical projection results from directed growth into new regions of the ICX, or by selective stabilization of an initially widespread but weak or non-functional projection, has not yet been resolved.

In either case, the factors that trigger such re-arrangements in connectivity have to operate at the level of the synapse. Adaptive modifications are experience-dependent, in other words, they are mediated by the electrical signals that neurons generate to communicate with each other. Since communication between neurons occurs at the synapses, it is not only natural but also necessary that changes begin here. Synapses 
have the capacity to detect patterns of activity and change their strength accordingly. The dominant model for activity-mediated modifications of neuronal circuits is based on Hebb and Stent's postulates of synaptic plasticity $[12,13]$. According to these, synapses are strengthened if presynaptic and postsynaptic activities are correlated and weakened if they are not. The likelihood of coordinating presynaptic and postsynaptic activities (and thus strengthening the synapse) is bound to increase if converging inputs are simultaneously active. The same principle could apply to converging visual and auditory inputs that signal events that are both seen and heard [5].

The favourite candidate for the molecular trigger of coincident activity is the $\mathrm{N}$-methylD-aspartate (NMDA) receptor, because of its dual dependency for activation on both glutamate (provided by activation of the presynaptic terminal) and depolarization (an indicator of postsynaptic activation). Evidence from a variety of systems, including the mammalian SC and the owl tectum, support the idea that these receptors are critically involved in the activity-mediated developmental refinements of neuronal connections. Specifically, chronic blockade of NMDA receptors in the SC prevents the normal development of auditory topography in the deeper layers, while having no effect on the visual map in the superficial layers [14]. Thus, the registration of the two maps is much less precise than in normal animals.

A critical role for NMDA receptors is also supported by experiments in prism-reared owls that were in the process of shifting their auditory spatial tuning. At intermediate stages in the shifting process, neurons respond strongly to both the normal range of ITDs and to the new, visually instructed range of ITDs. When NMDA receptors were pharmacologically blocked in the ICX of these animals, the newly adaptive responses were selectively suppressed compared to the normal ones, both in the tectum [15] and in the ICX [16]. This result indicates that NMDA receptors preferentially mediate the expression of newly learned responses and is reminiscent of findings in other systems showing a developmental progression of NMDA-to-AMPA dominance in excitatory connections [17]. Such data suggest that functional learning and acquisition of adaptive responses can result from synaptogenesis [18]. They also raise the question as to how the initial normal responses are eliminated during the process of re-tuning to the new visual coordinates. One possibility is that synapses mediating inappropriate (maladaptive) responses are weakened or eliminated through a process like long-term depression (LTD), as has been shown in other systems [19]. Alternatively, and non-exclusively, it is possible that the signals mediated by these synapses are selectively inhibited. Indeed, recent evidence suggests that this may be the case. Blocking inhibition at ICX sites that are expressing predominantly new, learned responses, results in the immediate re-appearance of strong normal responses [20]. This suggests that the circuitry necessary to support the normal (original) ITD maps can coexist with the newly acquired (learned) maps, but is actively suppressed by inhibition.

\section{Conclusion}

The structural and functional organization of neuronal circuits is to a large extent programmed genetically and is in place before the onset of sensory experience. These initial 
circuits, which are specific but less differentiated than in adult animals, can be thought as setting the limits for the range of functions the brain is able to support. At the same time, experience, in the form of neuronal activity generated by the neurons themselves, can modify and refine the characteristics of neurons and their assemblies and thus adapt them to the particular features and demands of the environment, as experienced by the individual. These activity-mediated modifications are based on synaptic plasticity (the ability of synapses to change their weight in a use-dependent fashion) and can involve both new growth and synaptogenesis as well as selective elimination of existing connections. The extent of this experience-dependent sculpting of the brain is greatest during a sensitive period early in life, but a lesser degree of adaptive plasticity is present throughout life, especially when animals are exposed to a rich sensory environment.

\section{Acknowledgements}

The author's work is supported by the Wellcome Trust.

\section{References}

[1] C.S. Goodman, Mechanisms and molecules that control growth cone guidance, Annu. Rev. Neurosci. 19 (1996) $341-377$.

[2] L.C. Katz, C.J. Shatz, Synaptic activity and the construction of cortical circuits, Science 274 (5290) (1996) $1133-1138$.

[3] B. Stein, M. Meredith, The Merging of the Senses, MIT Press, Cambridge, MA, 1993.

[4] Y.E. Cohen, E.I. Knudsen, Maps versus clusters: different representations of auditory space in the midbrain and forebrain, TINS 22 (3) (1999) 128-135.

[5] A.J. King, Sensory experience and the formation of a computational map of auditory space in the brain, BioEssays 21 (11) (1999) 900-911.

[6] E.I. Knudsen, Mechanisms of experience-dependent plasticity in the auditory localization pathway of the barn owl, J. Comp. Physiol., A 185 (4) (1999) 305-321.

[7] M.S. Brainard, E.I. Knudsen, Sensitive periods for visual calibration of the auditory space map in the barn owl optic tectum, J. Neurosci. 18 (10) (1998) 3929-3942.

[8] E.I. Knudsen, Capacity for plasticity in the adult owl auditory system expanded by juvenile experience [see comments], Science 279 (5356) (1998) 1531-1533.

[9] M.S. Brainard, E.I. Knudsen, Experience-dependent plasticity in the inferior colliculus: a site for visual calibration of the neural representation of auditory space in the barn owl, J. Neurosci. 13 (11) (1993) 45894608.

[10] A.J. King, J.W. Schnupp, I.D. Thompson, Signals from the superficial layers of the superior colliculus enable the development of the auditory space map in the deeper layers, J. Neurosci. 18 (22) (1998) 93949408.

[11] D.E. Feldman, E.I. Knudsen, An anatomical basis for visual calibration of the auditory space map in the barn owl's midbrain, J. Neurosci. 17 (17) (1997) 6820-6837.

[12] D. Hebb, The Organization of Behavior, Wiley, New York, 1949.

[13] G.S. Stent, A physiological mechanism for Hebb's postulate of learning, Proc. Natl. Acad. Sci. U. S. A. 70 (4) (1973) 997-1001.

[14] J.W. Schnupp, et al., NMDA-receptor antagonists disrupt the formation of the auditory space map in the mammalian superior colliculus, J. Neurosci. 15 (2) (1995) 1516-1531.

[15] D.E. Feldman, M.S. Brainard, E.I. Knudsen, Newly learned auditory responses mediated by NMDA receptors in the owl inferior colliculus, Science 271 (5248) (1996) 525-528. 
[16] D.E. Feldman, E.I. Knudsen, Pharmacological specialization of learned auditory responses in the inferior colliculus of the barn owl, J. Neurosci. 18 (8) (1998) 3073-3087.

[17] J.T. Isaac, R.A. Nicoll, R.C. Malenka, Silent glutamatergic synapses in the mammalian brain, Can. J. Physiol. Pharm. 77 (9) (1999) 735-737.

[18] D.E. Feldman, E.I. Knudsen, Experience-dependent plasticity and the maturation of glutamatergic synapses, Neuron 20 (6) (1998) 1067-1071.

[19] A. Artola, W. Singer, Long-term depression of excitatory synaptic transmission and its relationship to longterm potentiation, TINS 16 (11) (1993) 480-487.

[20] W. Zheng, E.I. Knudsen, Functional selection of adaptive auditory space map by GABAA-mediated inhibition [see comments], Science 284 (5416) (1999) 962-965. 